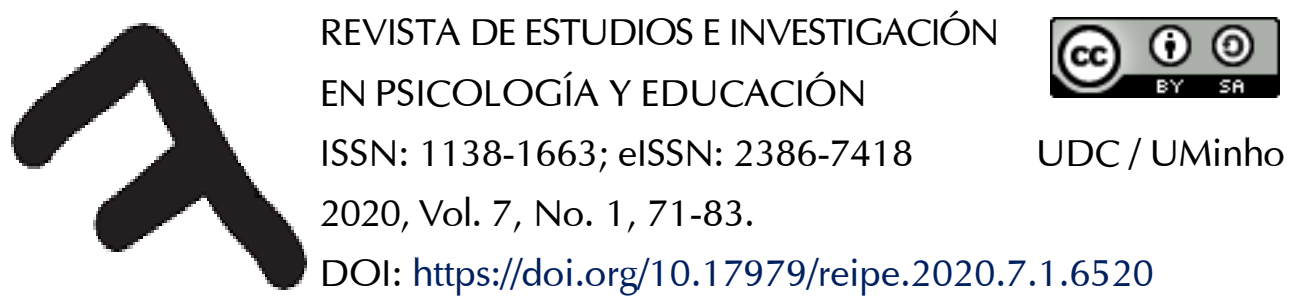

\title{
La grabación musical como herramienta de aprendizaje. Implicaciones educativas para el alumnado
}

\author{
Music recording as a learning tool. Educational implications for students
}

\author{
Aurelio Chao-Fernández (D), M Cristina Pérez-Crego (iD), Rocío Chao-Fernández (iD) \\ Universidade da Coruña
}

\begin{abstract}
Resumen
En la sociedad actual las TIC forman parte de nuestro entorno habitual. Por tanto, los procesos de enseñanza-aprendizaje no pueden mantenerse ajenos a esta realidad, debiendo adaptarse a los tiempos actuales, y aprovechar las posibilidades formativas que éstas ofrecen para formar ciudadanos competentes. En educación musical, a pesar de que existen múltiples herramientas digitales, como las grabaciones, constituyen un recurso infrautilizado. En esta investigación se pretende averiguar las implicaciones educativas que ofrece el uso de grabaciones al alumnado. Para ello se han realizado entrevistas a 41 docentes de música de conservatorios públicos, municipales y escuelas de música para conocer su visión sobre lo que aportan las grabaciones al aprendizaje musical. Los resultados indican que las grabaciones constituyen una eficaz herramienta de gran valor educativo, que se puede emplear desde la perspectiva de modelo a seguir, como de autocorrección cuando es el mismo estudiante quien graba su interpretación. Se concluye que además suponen un excelente recurso motivacional y creativo y que gracias a ellas se mejora la autoescucha, la autoconsciencia, la autocrítica y autoevaluación de la propia exposición auditiva.
\end{abstract}

Palabras clave: grabaciones musicales; aprendizaje; TIC; música

\begin{abstract}
ICT has become an integral part of everyday life. Education cannot ignore this reality but must instead move with the times and take advantage of the educational potential of technology to provide students with the tools to become competent citizens. In music education, digital tools, such as recordings, are an underused resource. The aim of this study is to assess the teaching-learning implications of digital music recording for students. Interviews were conducted with 41 music teachers from public, municipal and private music schools to find out their opinion about the contribution of digital recordings to music learning. The results confirm the educational value and effectiveness of recordings, both as a model for students to follow and as a method of self-correction. The study concludes that recordings are also an excellent motivational and creative resource and a way to improve students' self-listening, self-awareness, selfcriticism and assessment of their own aural exposure.
\end{abstract}

Keywords: music recordings; learning; ICT; music

Aurelio Chao-Fernández (Dorcid.org/0000-0001-7846-7637, Ma Cristina Pérez-Crego Đorcid.org/0000-0001-5937-0047 y Rocío ChaoFernández Dorcid.org/0000-0002-6584-0049: Departamento de Didácticas Específicas y Métodos de Investigación y Diagnóstico en Educación, Facultad de Ciencias de la Educación, Universidade da Coruña. Campus de Elviña s/n, 15071 A Coruña, España.

Correspondencia relativa a este artículo: Aurelio Chao-Fernández - aurelio.chao@udc.es 
La educación debe estar en consonancia con los continuos cambios que se producen incesantemente a nivel social, cultural y profesional (Belloch, 2012), muchos de ellos relacionados con las Nuevas Tecnologías de la Información y la Comunicación (en lo sucesivo, TIC). Efectivamente, a inicios de milenio muchos países estaban comprometidos con las nuevas tecnologías para mejorar la calidad y la eficacia del aprendizaje escolar (Ministerio de Educación, Cultura y Deporte, 2001), pero requieren un uso y conocimiento adecuados.

Estos cambios implicaron que paulatinamente muchas aulas fuesen dotadas con recursos tecnológicos como ordenadores, proyectores, tabletas digitales... intentando adaptarse al nuevo tipo de sociedad: la sociedad de la información (Adell y Castañeda, 2012). Totalmente necesario si la educación quiere estar en consonancia con la realidad, pues gran parte del alumnado tiene en su casa o en su entorno más próximo algún recurso tecnológico, lo que además provoca que asimilen de forma natural la aparición de las nuevas tecnologías en el aula (Marqués, 2012).

Evidentemente la escuela debe favorecer que las TIC se integren en las aulas, ya que benefician la alfabetización digital, son fuente de información, facilitan los procesos de enseñanza-aprendizaje, rompen las barreras espacio-temporales, son elementos motivadores y promueven la creatividad (Belloch, 2012; Ferro, Martínez y Otero, 2009; Marqués, 2012; Román, Chao-Fernández y Mato-Vázquez, 2018). Indudablemente, el avance tecnológico en su compromiso de rediseñar el acto educativo hacia una educación 2.0, provoca que dentro de los diferentes entornos educativos surjan nuevos espacios de aprendizaje y replanteamientos en la pedagogía musical (Cabero y Barroso, 2013; Area y Ribeiro, 2012), pero el docente debe ser consciente de que, si las TIC no se emplean adecuadamente, no serán efectivas (Díez y Carrera, 2018; Valcárcel y González, 2013).

Las TIC implican también cambios y transformaciones en la enseñanza de la música (Arques e Navas, 2012), pero su introducción no debe verse como algo invasivo, que trata de eliminar los métodos existentes, sino para defenderlos y enriquecerlos, ya que existe una gran cantidad de contenido que se puede trabajar a través de las TIC y, por lo tanto, beneficiarse de él. El aprendizaje auditivo, y las interpretaciones, por ejemplo, se pueden optimizar a través de multimedia. El hecho de que haya nuevas tecnologías en el aula de música puede conseguir que aumente la motivación, la creatividad y el rendimiento académico de los estudiantes (Carrera, Román y Chao-Fernández, 2017). Además, según estas autoras, no podemos olvidar que las aulas de música están ligadas desde siempre a la tecnología: equipos de música, cámaras de vídeo, grabadoras, CDs... materiales que se emplean para favorecer el trabajo del profesorado y enriquecer sus prácticas.

Por su parte los centros educativos como Conservatorios y Escuelas de música también tratan de adaptarse progresivamente a estos nuevos tiempos, incorporando estos recursos a sus proyectos curriculares y educativos (Gisbert, 2018).

Hay dos acontecimientos que transformaron la difusión de la música: por una parte, la partitura impresa, con lo cual la posibilidad de interpretación de una obra musical se expandió, especialmente en Europa, alcanzando un mercado donde el manuscrito no podía llegar. En segundo lugar y quizás con la misma importancia (Clarke, 2006), fue la aparición de la grabación, hace aproximadamente 160 años, llegando de una manera globalizada tanto la música de un compositor, como la versión de los diversos intérpretes, a todos los rincones del planeta (Pérez Sánchez, 2012). Incluso con la aparición de las grabaciones hay quien otorga más atención a la responsabilidad del intérprete que a la propia obra musical. Es en este momento cuando se puede estimar la partitura como un elemento diferente al de la grabación, restándole importancia a la partitura, al considerarla como un proyecto y a la grabación como su realización. Efectivamente, la invención del fonógrafo permitió realizar estudios empíricos sobre la interpretación musical, algo que anteriormente era inviable, lo cual otorga mayor protagonismo al intérprete, quedando la partitura como un mero elemento del proceso de comunicación (Gértrudix Barrio, 2003).

Indudablemente las grabaciones sonoras aportan un valor extraordinario a la formación musical, que se debe analizar desde varias perspectivas. En primer lugar, se puede considerar como un documento sonoro de los artistas e intérpretes que nos precedieron, y que nos legan para la posteridad, las cuales puede utilizar un estudiante como modelo a seguir. 
Hasta el momento de la producción industrial de los discos, la forma de estudio y análisis de una interpretación solamente era posible realizarla en vivo, por lo que eran irrepetibles, pero gracias a las grabaciones podemos escuchar una y otra vez la misma obra, ejecutada incluso por diferentes músicos, con lo cual podemos sacar ideas y conclusiones para nuestra propia interpretación, tratando así las grabaciones como documentos (Clarke, 2006). Además, según Miranda Regojo (1990), la grabación:

Hace hoy posible a los musicólogos la realización de investigaciones, no sólo tomando como base una partitura única sino toda una serie de versiones de una misma obra ejecutadas por distintos intérpretes o por idéntico intérprete en distintas ocasiones, así como el estudio de la evolución de la técnica de los grandes intérpretes a partir de todos los trabajos por aquél grabados a lo largo de su carrera (p.31).

Otra de las repercusiones de la aparición de las grabaciones es que gracias a ellas es posible un estudio y un análisis más subjetivo de la música. Al ser sus reproducciones iguales unas a otras, en contraste con las interpretaciones realizadas en vivo, se pueden realizar comparaciones midiendo parámetros como la altura, intensidad, timbre, frecuencia, etc. naciendo así la musicología empírica. Por otro lado, al poder reproducir la música en cualquier sitio, provoca que ya no esté ligada a ser escuchada en las salas de conciertos, provocando que pueda ser oída en cualquier lugar.

Por último, la grabación sirve, según Arthur Rubinstein (citado por Carrascosa y Ruiz-Silva, 1977), como recurso para la revisión de cómo ha acometido el discente el estudio de una obra musical:

El disco es "el profesor ideal", refiriéndose a la posibilidad que ofrece de escucharse a sí mismo, y así poder corregir los defectos, ya que, mientras toca, el ejecutante no oye lo que está tocando con la independencia y el distanciamiento precisos para juzgarlo con objetividad. Y no digamos si se confronta una grabación propia con la de otros colegas (p.121-122).

Atendiendo a Chao-Fernández, Pérez Crego y García Armas (2019) la formación de un instrumentista se divide en diferentes partes, siendo la interpretativa una de las más importantes. Tradicionalmente ésta se apoyaba en las explicaciones del docente, o a la asistencia a conciertos, pero en la actualidad encuentra en las grabaciones un extraordinario complemento:

El recurso de las grabaciones resulta el más interesante y completo que existe en nuestros días. Es muy habitual que un maestro les indique a sus alumnos que traten de localizar grabaciones con diferentes versiones de las obras que están estudiando para así, obtener un gran abanico de posibilidades interpretativas (p. 2004).

En esta línea De Castro (2015) realizó una investigación sobre los beneficios de los recursos educativos TIC, y concretamente la grabación, dentro de la enseñanza musical pianística:

El objetivo fundamental de esta actividad fue detectar "cómo estudia el alumno", qué mecanismos utiliza y qué tipo de respuestas manifiesta. Con esta vídeo-grabación se pretendió registrar los primeros momentos de acercamiento a una pieza/s nueva/s, ya que la propuesta planteada constaba de obras no conocidas por el grupo de alumnos, desde un punto de vista técnico. Con ello se procedió después a observar las acciones, conductas y actitudes que se manifestaron a lo largo del período de grabación, en definitiva, una situación de estudio en un contexto real y definido (p. 41).

Todo lo expuesto convierte a las grabaciones en un valioso material digital de empleo en el aula de música, tanto en formato de audio como de vídeo. En lo referente a las primeras, aportan una gran utilidad pues permite desarrollar una audición crítica, mientras que con el vídeo podemos observar con detalle ciertos aspectos de la ejecución que de otro modo podrían pasar inadvertidos. En todo caso, el uso de cualquiera de las dos puede ser beneficioso tanto desde la perspectiva creativa como interpretativa, además de constituir una excelente ayuda para el desarrollo de una serie de habilidades musicales como las auditivas, visuales y motrices. Según Alfred Brendel (2013) la grabación está entre los requisitos para aprender a tocar un instrumento:

Disponer de un buen instrumento; una buena edición del texto original; una técnica interpretativa que no ocasione ningún daño físico al pianista; trabajo concentrado, pero no 
encarnizado; ambición, paciencia; la elección de las piezas adecuadas; y en último lugar, pero no por ello menos importante, la facultad de escucharse a sí mismo durante la ejecución. Esta facultad habrá que ir desarrollándose en el transcurso del tiempo y con ayuda de una grabadora. Cuando cumplí veinte años, unas personas muy amables me regalaron un magnetófono Revos, todavía lo conservo (p. 102).

El estudio que se presenta forma parte de una amplia investigación sobre las grabaciones que realiza la CRTVG. Los datos de ese estudio, llevado a cabo con metodología cuantitativa, pusieron de manifiesto la necesidad de profundizar en las posibles implicaciones educativas que el recurso de las grabaciones puede ofrecer al alumnado. Por ello, debido a la relevancia del tema se ha optado por abordarlo desde una óptima metodológica cualitativa para comprender, analizar e interpretar el significado que los participantes del fenómeno de estudio, los docentes de educación musical, muestran a partir de sus propios discursos y reflexiones. Para, de esta manera, dar respuesta a la pregunta de investigación: ¿Qué implicaciones tiene para el alumnado el uso de grabaciones como herramienta educativa?

\section{Método}

\section{Participantes}

El estudio recoge los testimonios de 41 docentes, de tres tipos de centros de formación musical: conservatorio público, conservatorio municipal y escuelas de música, tal y como se puede observar en la Figura 1. Cabe destacar que en el caso de los centros públicos la muestra representa la totalidad de los conservatorios existentes en la Comunidad Autónoma de Galicia.

\section{Figura 1}

Tipos de centro de formación musical

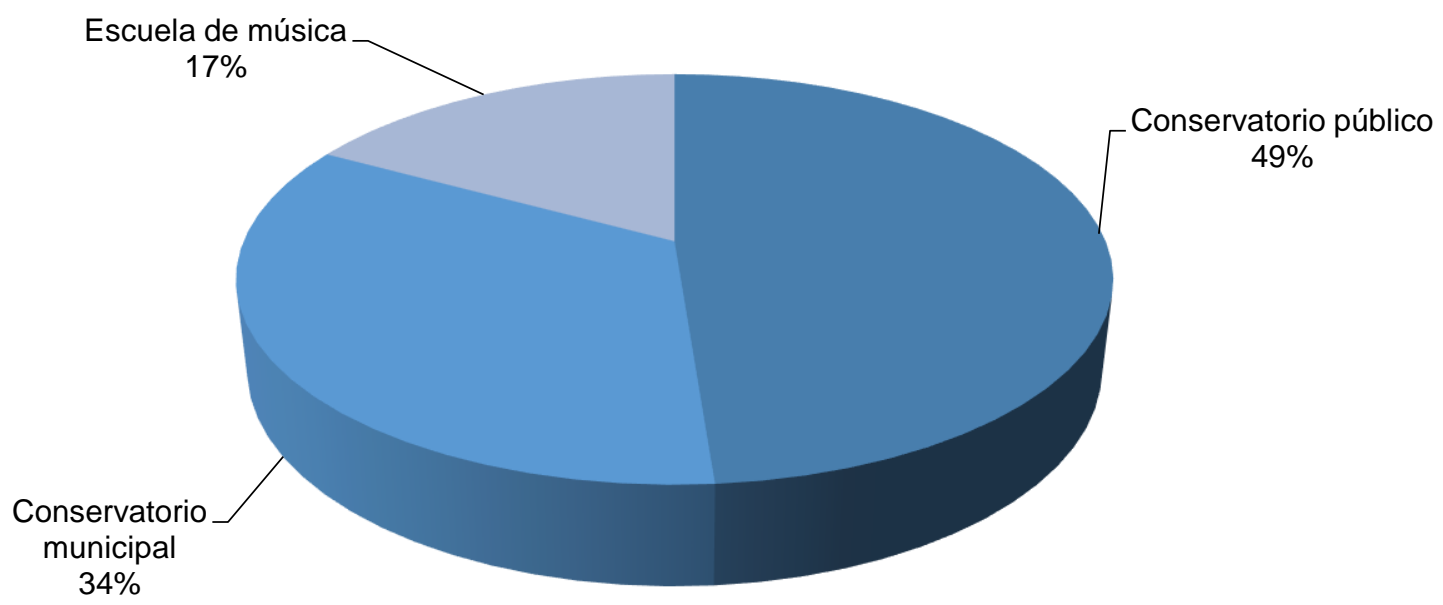

La muestra recoge testimonios de informantes con un amplio abanico de trayectorias profesionales en la enseñanza musical, tal y como se muestra en la Figura 2, siendo mayoritaria la franja entre 11 y 15 años de experiencia, y minoritaria la de más de 20 años de experiencia. Cabe señalar que existe un equilibrio entre los docentes con experiencia entre 1 y 5 años y 6 y 10, porcentaje similar también, aunque ligeramente más elevado, de los docentes con experiencia entre 16 y 20 años. Para con ello poder obtener una visión más amplia de las posibilidades del recurso de las grabaciones en el ámbito educativo, a través del relato de docentes con diversas vivencias educativas.

Además, la muestra está compuesta por una representación de las diferentes especialidades instrumentales, siendo mayoritaria la representación de piano, clarinete, guitarra, percusión y violín, tal y como se observa en la Figura 3, y minoritaria la presencia de docentes de armonía, canto, flauta, gaita, trombón, trompeta y violonchelo. 


\section{Figura 2}

Años de experiencia profesional de los informantes

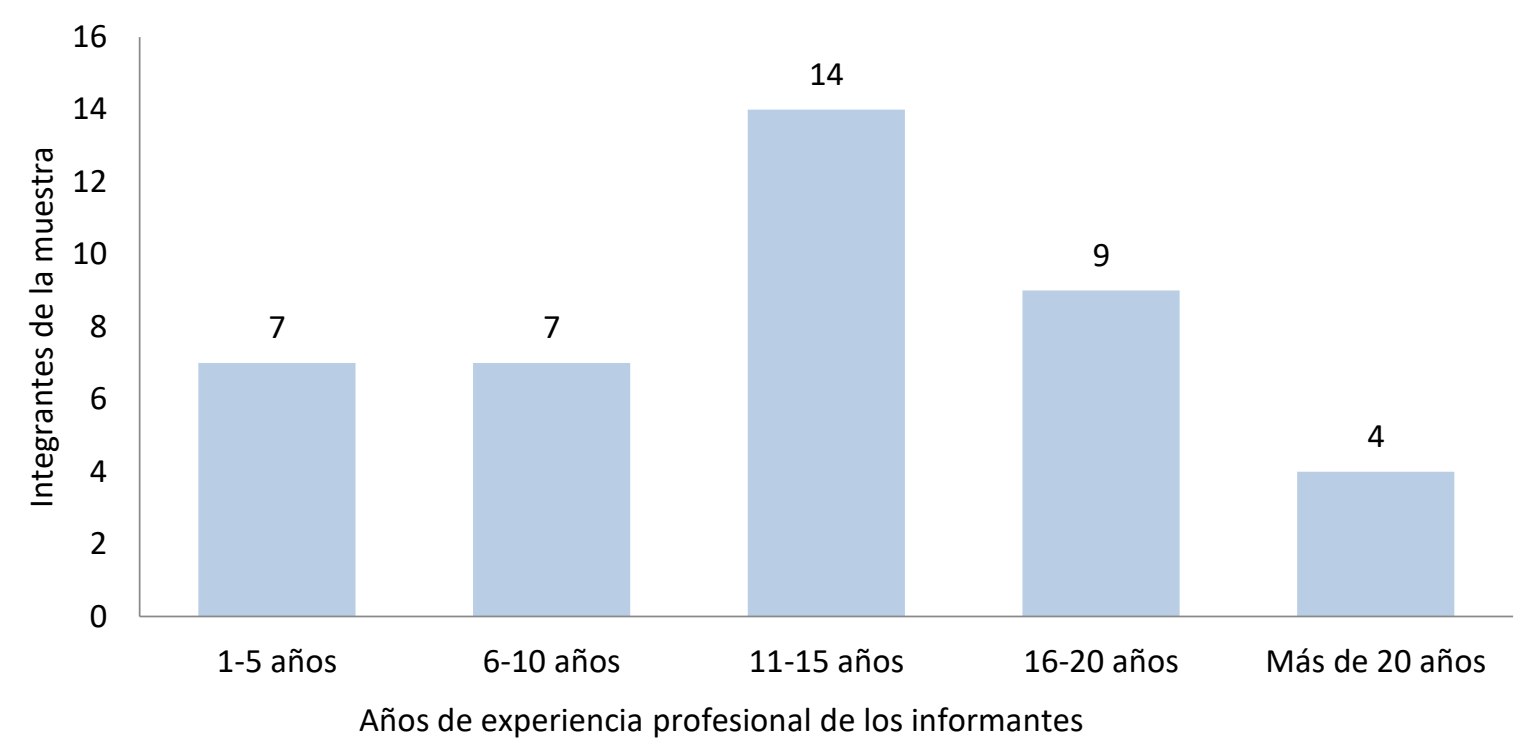

\section{Figura 3}

Especialidades instrumentales de la muestra

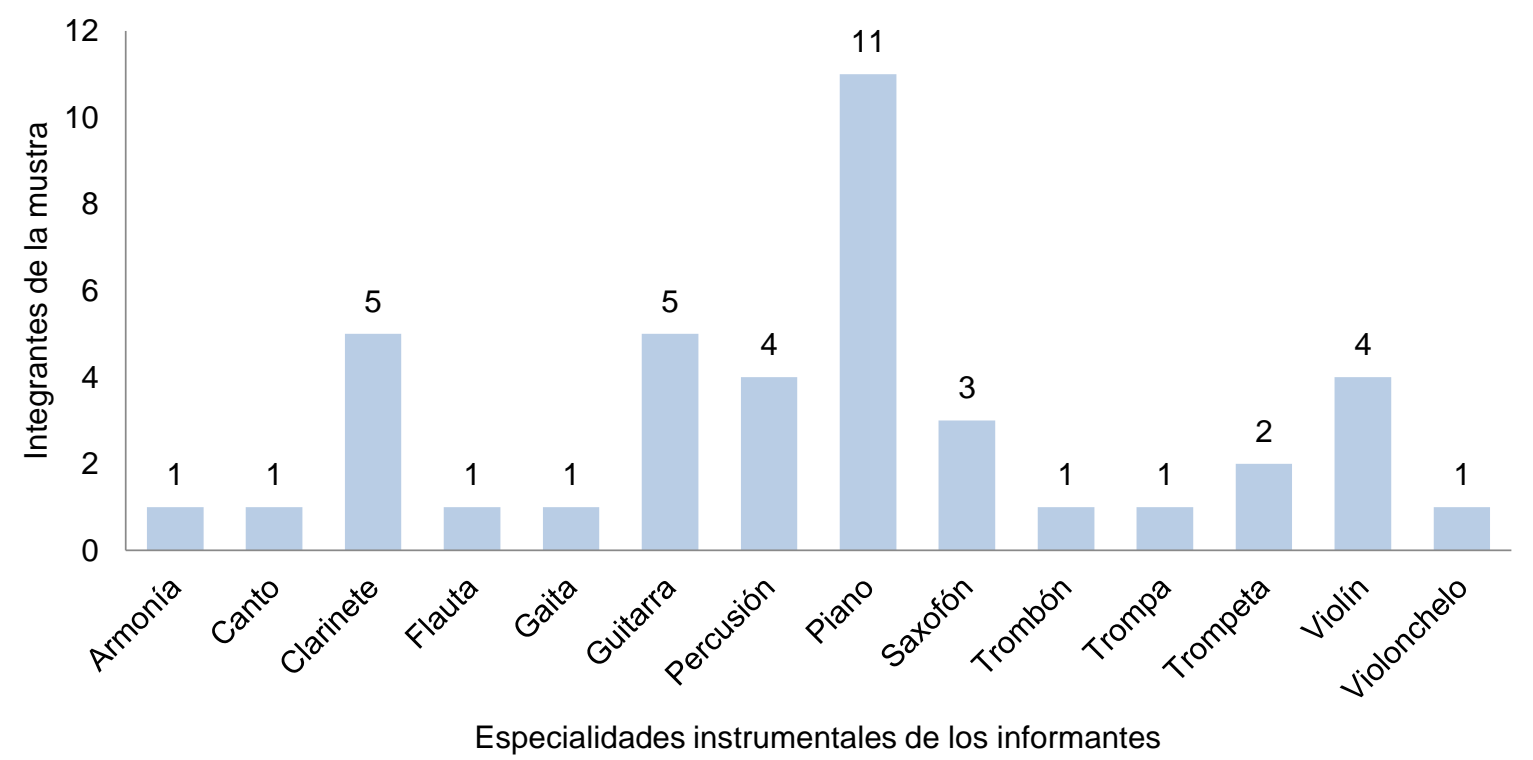

La negociación de condiciones para la recogida de información se estableció al comienzo del proceso. Mediante un acuerdo verbal (Stake, 1998), se explicaron los propósitos de la investigación (conocer las implicaciones que tiene para el alumnado el uso de grabaciones como herramienta educativa). Con respecto al desarrollo del estudio las cuestiones acordadas con los participantes versaron sobre la recogida y tratamiento de la información. Los docentes aceptaron el uso de la entrevista como técnica de recogida de datos, con el apoyo de una grabadora como recurso técnico para facilitar de esta manera la posterior transcripción de la información obtenida y la validación de la misma por parte de las personas entrevistadas. Para comodidad de los informantes, las entrevistas fueron realizadas en los lugares y espacios seleccionados por los profesionales 
para facilitar una mayor familiaridad en la recogida de datos. Para respetar el anonimato de los docentes se realizó mediante la codificación de la información identificativa de los mismos. Asimismo, se llegó al acuerdo de que los datos solo tendrían fines de investigación, siendo los validados los únicos que se utilizarán en la elaboración del texto. Manteniendo una estructuración e interpretación de los datos de una forma rigurosa y científica facilitando el acceso a los mismos a los participantes, si así lo considerasen oportuno.

\section{Instrumento}

La técnica seleccionada para el recabado de la información ha sido la entrevista semi estructurada. La recogida de datos se llevó a cabo en los diferentes centros de formación musical de los profesionales entrevistados. El registro se realizó de una manera fidedigna y respetando el criterio de literalidad estilística (Pujadas, 2000), que nos ha permitido mantener la rigurosidad de la información obtenida. Además, se ha seguido un proceso de validación de la información por parte de los informantes mediante feedback de los participantes.

\section{Procedimiento}

El diseño y desarrollo del estudio ha partido de un diseño circular abierto, para poder reflexionar permanentemente sobre el proceso de investigación, tomando como referente "el modelo circular de investigación de Glasser y Strauss" (citado en Flick, 2004, p.59). La finalidad que se persigue con el empleo este diseño, es captar el significado que tienen para los sujetos, sus propias acciones (Stake, 1998). Este diseño permite ver cómo los supuestos teóricos se vuelven relevantes a partir de las versiones preliminares que tienen los participantes sobre el conocimiento del objeto de estudio y sobre las perspectivas que tienen sobre el mismo, las cuales se van reformulando y elaborando durante el proceso de investigación. A medida que se van recogiendo datos se analizan y se van construyendo y reconstruyendo los supuestos teóricos sobre el análisis de los mismos de manera continua. Es un proceso de recolección de datos y análisis sistemático en relación recíproca mediante un entrelazamiento circular de pasos empíricos.

Con respecto a la ética del proceso de investigación, se ha llevado a cabo un riguroso y respetuoso tratamiento de los datos. Los criterios de credibilidad con los que se ha desarrollado la investigación responden a tres cuestiones: la fiabilidad, la validez y la triangulación de datos (Stake, 1998). La fiabilidad está fundamentada en la obtención y registro de los datos de una manera clara (Flick, 2004), e indicando cuáles son las declaraciones de los informantes (información entre comillas con un código identificador). Así mismo se explica el proceso de codificación de los datos dejando constancia de las categorías y códigos obtenidos en el análisis. La validez ha sido abordada con la validez comunicativa por parte de los informantes (Flick, 2004) y la validación de la información a través de los sujetos investigados. Por último, la triangulación de datos (Denzin, 1989), se sustenta mediante el uso de diferentes datos de distintos informantes.

\section{Análisis de los datos}

En el proceso de recogida de información se fueron interpretando los datos y reformulando los conceptos teóricos a medida que avanzaba el estudio. Posteriormente se realizó un análisis mediante un proceso inductivo de categorización de la información (Miles, Huberman, y Saldaña, 2014; Miles y Huberman, 1994) para "reconstruir las categorías específicas que los participantes emplean en la conceptualización de sus propias experiencias" (Goetz, y Lecompte, 1988 p.31) y con ello poder comprender a partir de sus propias percepciones cuáles son las implicaciones educativas que el recurso ofrece. A partir del proceso de codificación de los datos, las categorías y códigos obtenidos en el análisis se presentan en la Tabla 1.

\section{Resultados}

Los datos revelan que el uso de las grabaciones en el ámbito educativo constituye un eficaz y valioso recurso en el proceso de aprendizaje del alumnado. Los resultados están sostenidos en los siguientes cuatro ejes. Las grabaciones como: 
1. Herramienta de apoyo de estudio técnico, sonoro e interpretativo.

2. Recurso para la mejora de las habilidades del alumnado.

3. Medio de aprendizaje relevante y vivencial (acercamiento a la realidad profesional).

4. Herramienta de mejora de la calidad educativa (recurso para la mejora de la interpretación musical).

A continuación, se exponen los resultados del estudio tomando como referencia los testimonios de los informantes recogidos en las entrevistas.

\section{Tabla 1}

Sistema de códigos y categorías de estudio

\begin{tabular}{|c|c|}
\hline Categoría & Códigos \\
\hline Aspectos formación musical & $\begin{array}{l}\text { Técnicos } \\
\text { Sonoros } \\
\text { Interpretativos } \\
\text { Visuales } \\
\end{array}$ \\
\hline Desarrollo de actitudes/habilidades & $\begin{array}{l}\text { Autoconsciencia } \\
\text { Autoescucha } \\
\text { Autoevaluación } \\
\text { Autocorrección }\end{array}$ \\
\hline Motivación (recurso motivacional) & $\begin{array}{l}\text { Dimensión lúdica } \\
\text { Meta } \\
\text { Incentivo } \\
\text { Satisfacción personal } \\
\text { Compromiso emocional }\end{array}$ \\
\hline Colaboración/ Trabajo en equipo & $\begin{array}{l}\text { Grado de implicación } \\
\text { Trabajo colaborativo } \\
\text { Compañerismo }\end{array}$ \\
\hline Herramienta de aprendizaje & $\begin{array}{l}\text { Mejora del estudio } \\
\text { Lado analítico y crítico } \\
\text { Madurez interpretativa } \\
\text { Herramienta grabación } \\
\text { Concentración } \\
\text { Instrumento de evaluación docente } \\
\text { Pedagógicamente positivo }\end{array}$ \\
\hline Aprendizaje relevante (vivencial) & $\begin{array}{l}\text { Espectadores de ellos mismos } \\
\text { Comparación (lo que creemos que suena y lo que suena realmente) } \\
\text { Crítica constructiva } \\
\text { Visión musical obra } \\
\text { Acercamiento realidad profesional } \\
\text { Utilidad formación } \\
\text { Parte interpretativa } \\
\text { Promoción } \\
\text { Experiencia musical } \\
\text { Transcendencia aprendizaje } \\
\text { Experimentación proceso grabación } \\
\text { Herramienta ayuda para otros } \\
\text { Valor de la grabación (en el tiempo) }\end{array}$ \\
\hline Herramienta de calidad educativa & $\begin{array}{l}\text { Calidad interpretativa } \\
\text { Exigencia interpretación } \\
\text { Recurso de grabaciones de calidad } \\
\text { Incidencia práctica educativa } \\
\text { Mejora rendimiento } \\
\text { Detección de errores } \\
\text { Perfección obra } \\
\text { Ajuste parámetros (técnicos y acústicos) }\end{array}$ \\
\hline
\end{tabular}




\section{Herramienta de apoyo de estudio técnico, sonoro e interpretativo}

En los discursos de los docentes se puede observar que el uso de grabaciones nos permite trabajar los contenidos que "forman parte del aprendizaje técnico, musical, interpretativo y social de alumno" (D34, CM, 27:9), siendo estos claves en el aprendizaje de la enseñanza musical de alumnado.

Las grabaciones como recurso en el aula, "permite conocer las diferentes técnicas de grabación y preparación de una grabación" (D11, CP, 3:11). El estudio de una obra supone abordar varias fases, tal y como podemos ver en la figura 4. Cada una de ellas mantiene una interconexión con la siguiente, la cual permite el perfeccionamiento del estudio del alumnado en cada paso que se da en su aprendizaje.

\section{Figura 4}

Fases por la que pasa el estudio de una obra.

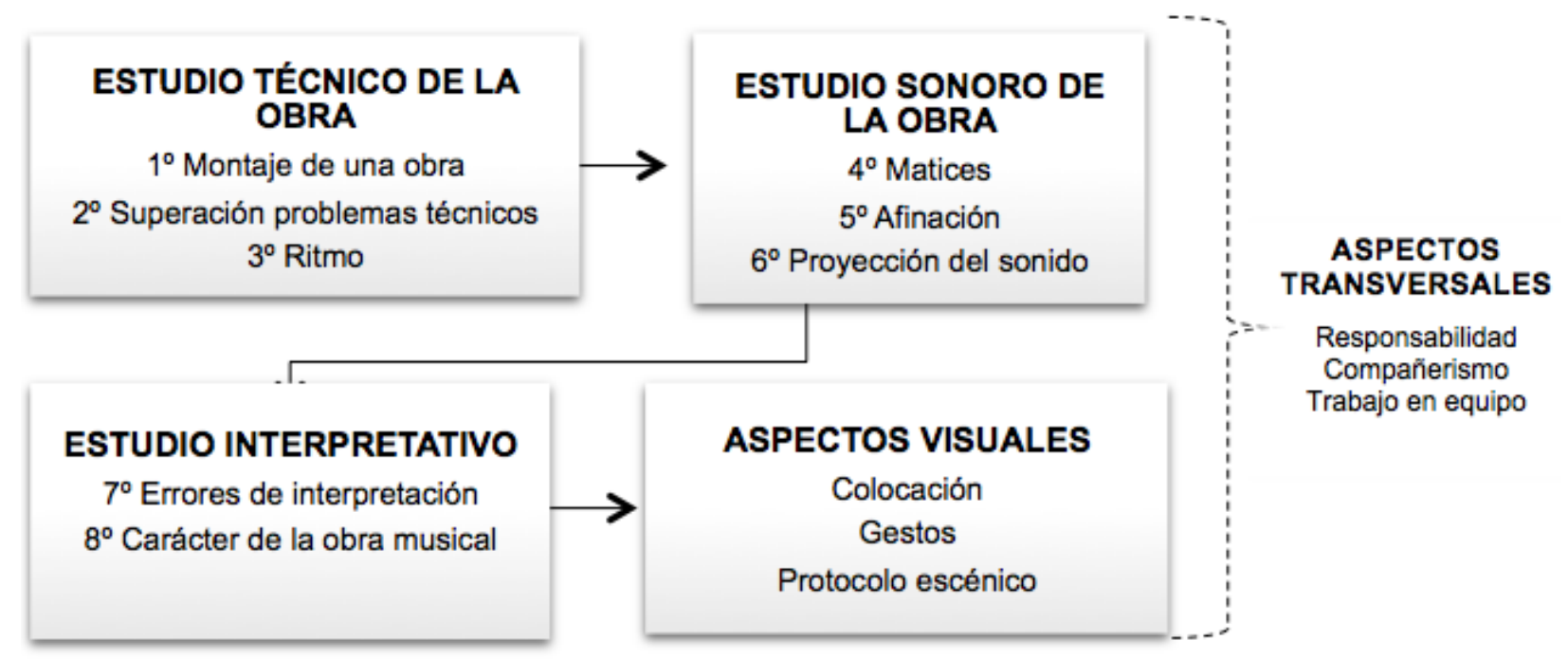

Las grabaciones "forman parte del aprendizaje técnico" (D34, CM, 27:10), y ayudan a poner en práctica cuestiones como, "focalizar lo aprendido para lograr un objetivo: el de la grabación. En el caso de instrumentos amplificados, también ayuda a ajustar mejor muchos parámetros técnicos y acústicos que frecuentemente se soslayan" (D39, EM, 32:10).

De hecho, el "acceder a una grabación profesional no es siempre fácil, con lo cual el alumno debe prepararse para ese momento de una manera especial en cuanto a precisión en la afinación y el ritmo, concentración, proyección del sonido, matices, etc." (D19, CP, 43:8). Son por lo tanto un recurso en el estudio técnico y sonoro de una obra, el cual permite trabajar cuestiones como la altura, timbre, frecuencia, intensidad, etc. "Los beneficios son de diversa índole, ... aquellos que forman parte del aprendizaje de la técnica derivada del montaje de una obra, afinación, superación de problemas técnicos" (D33, CM, 26:10).

Así mismo, "las grabaciones te permiten corregir aspectos visuales como colocación, gestos, protocolo escénico, errores de interpretación, carácter de la obra musical, comunicación con el público, etc.” (D14, CP, 6:9). Siendo una herramienta para estudio interpretativo de la obra, además de trabajar cuestiones visuales de la interpretación.

Aportando además varios beneficios al alumnado, como puede ser un buen recurso para trabajar "aquellos aspectos más transversales como son la responsabilidad, el compañerismo, el trabajo en equipo, etc." (D33, $\mathrm{CM}, 26: 10)$.

\section{Recurso para la mejora de las habilidades del alumnado}

Los informantes consideran que la grabación es un recurso que debe tomarse en consideración en la práctica cotidiana docente de la educación musical, ya que:

1. Mejora la habilidad del estudiante en el proceso de la formación musical: "La grabación es una 
herramienta fundamental para mejorar en el estudio" (D18, CP, 10:8), "las grabaciones ayudan al alumno a mejorar" (D27, CM, 19:8). Así como "les obliga a adquirir hábitos de estudio positivos para realizar las grabaciones" (D36, EM, 29:9).

2. Favorece el proceso de autoescucha y de educar el oído: Desarrolla por lo tanto la facultad de escucharse a sí mismo en la ejecución, siendo por lo tanto una "herramienta fundamental para la autoescucha, y ayuda a concretar los aspectos técnicos y musicales que tiene que mejorar el alumno en el estudio" (D18, CP, 10:10).

3. Ofrece además una oportunidad de auto examinarse, de valorarse a sí mismos: "Pienso que eso puede ayudar a los propios alumnos a valorarse viéndose desde el otro lado. Algunas veces grabo a los alumnos para que posteriormente se vean y se escuchen y objetivamente saquen sus propias conclusiones del trabajo realizado e intenten encontrar cómo mejorar ciertos aspectos" (D26, CM, 18:8).

4. Permite la autocorrección al discente, la posibilidad de corregir errores: "El que los alumnos realicen grabaciones de sus propias interpretaciones siempre es positivo ya que por una parte se les obliga a tener una interpretación lo más correcta posible y el escuchar sus propias grabaciones les ayuda a autocorregirse" (D5, CP, 37:8). "Deben esforzarse más a la hora de tocar la obra lo más perfectamente posible y el escuchar tu propia interpretación nos ayuda a corregir posibles defectos" (D9, CP, 37:9).

5. Fomenta la capacidad de análisis y crítica: "Agudiza su lado analítico y crítico, ayudando a la madurez interpretativa" (D29, CM, 21:9). Siendo un medio que permite "hacer autocrítica" (D12, CP, 4:11) del trabajo realizado.

6. Favorece la concentración en la interpretación de una obra: "Los beneficios son múltiples, como dije antes está la preparación que es diferente a la de una clase, ya que en ésta se puede parar de tocar y en un concierto o una grabación no, y eso supone una diferencia abismal en el tipo de concentración" (D19, CP, 43:10).

\section{Medio de aprendizaje relevante y vivencial (acercamiento a la realidad profesional)}

A través de la grabación, el alumnado vivencia de primera mano cómo se percibe realmente la interpretación realizada. El recurso permite discernir autónomamente los posibles errores cometidos en la interpretación: "la grabación puede funcionar como referencia para comprender cómo se nos percibe tocando desde fuera. Además, es una buena herramienta para darse cuenta de forma autónoma de ciertos errores o imperfecciones que pueden resultar difíciles de percibir durante la práctica" (D24, CM, 16:9). Esto es debido a que el intérprete que ejecuta la obra no oye lo que está tocando con objetividad suficiente. "También se benefician del análisis posterior, ya que los músicos tendemos a enfatizar la escucha subjetiva (lo que oímos en nuestra cabeza mientras tocamos) y necesitamos la grabación para ver las diferencias entre lo que creemos que sonaba y lo que en realidad sonaba, de esta manera nos permite hacer los cambios necesarios para que esa diferencia se vaya reduciendo" (D19, CP, 43:11). Por lo que la grabación ofrece la posibilidad de la reproducción de la obra interpretada para poder comprender la interpretación real de la misma, "son espectadores de ellos mismos, la perspectiva cambia. Puede desarrollar la crítica constructiva, darse cuenta de lo que hacen bien y no tan bien" (D3, CP, 22:9).

Varios informantes consideran las grabaciones como un buen recurso para visibilizar el trabajo realizado en el aula y poder ser evaluado, "si la grabación es directamente al alumnado, puede ser un instrumento más de evaluación por parte del profesor y también de autoevaluación por parte del alumno. Si la grabación es externa, proporciona otra visión musical de lo que se está trabajando" (D40, EM, 34:9), "creo que sí se deben realizar, porque es una muy buena herramienta para ellos el hecho de preparar las obras para grabarse, escucharse posteriormente y experimentar el hecho propio de la realización de una grabación" (D4, CP, 33:9). "Son una buena forma de visibilizar el trabajo hecho en clase y a su vez, sirven para que el alumnado adquiera experiencia frente a futuras grabaciones" (D41, EM, 35:8).

Además de ser un medio de acercamiento a la futura realidad profesional, rompiendo así la barrera entre el aula y la futura práctica profesional ya que "ayudan al desarrollo del alumno, así como complementan el trabajo fuera del aula, y potencian la exposición ante un público" (D22, CM, 14:8). "Al mismo tiempo que se autoexaminan y se escuchan desde fuera de su propio instrumento, tienen ese tiempo de grabación en el cual deben de estar concentrados poniéndose en situación de audición, concierto, examen y prueba, lo cual también 
les sirve para un futuro en el cual tener trabajado ese aspecto es importante" (D26, CM, 18:9), por lo que "se familiariza con el mundo de las grabaciones artísticas" (D27, CM, 19:9).

Otra de las ventajas del recurso de las grabaciones digitales es la posibilidad de tener material promocional del propio alumno, "permite tener material promocional y de calidad" (D11, CP, 3:13), "siendo un método de exposición de su trabajo" (D22, CM, 14:9).

La posibilidad de grabar la interpretación de cada uno, tiene además un carácter motivador, "puede ser algo motivador para ellos y al mismo tiempo es una herramienta para que se escuchen y aprendan a través del hecho de grabarse y escucharse" (D4, CP, 33:11), "puede aportar un plus de motivación, así como un conocimiento más amplio del trabajo que se hace" (D9, CP, 41:9). Siendo además "importante para los alumnos ver que lo que aprenden a diario tiene un objetivo, una trascendencia más allá del aula” (D38, EM, 31:9).

Así como "les ayuda a tener objetivos y a conocer mediante la práctica algo que es habitual entre los músicos profesionales" (D2, CP, 11:9), siendo una buena oportunidad "para desarrollar su experiencia musical" (D31, $\mathrm{CM}, 24: 8)$.

Además, "la propia grabación puede servir posteriormente de herramienta de ayuda a otros alumnos" (D4, $\mathrm{CP}, 33: 10$ ), porque las grabaciones "ayudan a escuchar a otros músicos, a aprender de ellos y corregir errores" (D38, EM, 31:8). Esto es debido a que nos permite poder tener acceso a versiones de una misma obra ejecutada por diferentes intérpretes. Por lo que es relevante "fomentarlas, es necesario dar a conocer su existencia y facilitar el acceso a las mismas" (D8, CP, 40:8).

En definitiva, ayuda a tener consciencia completa del sonido en el tiempo: "Ya que la música solo tiene sentido en la mente del receptor en el momento exacto en el que se está produciendo, cualquier grabación ayuda a darle importancia en el tiempo" (D40, EM, 34:8). Teniendo además la oportunidad de crear con ellas "una videoteca para el centro educativo" (D35, EM, 28:8).

\section{Herramienta de mejora de la calidad educativa (recurso para la mejora de la interpretación musical)}

Los participantes del estudio indican con sus relatos que las grabaciones suponen un recurso favorecedor de la calidad educativa en el aprendizaje de la educación musical. Promoviendo la autonomía del alumnado y perfeccionando la capacidad de expresión musical con el fin de alcanzar una calidad artística. "Es un recurso educativo muy útil para la mejora en la interpretación musical por la autoconsciencia por parte del alumno/a" (D1, CP, 1:9). Además "beneficia el conocimiento del proceso de grabación que ayudará a desarrollar mejor este tipo de actividades de cara al futuro" (D25, CM, 17:9).

Esta herramienta permite el ajuste de parámetros técnicos y acústicos, "para mayor conocimiento del repertorio; para descubrir los pormenores y dificultades técnicas de su realización; para conocer el grado de implicación y preparación previa que requieren grabaciones de este tipo" (D16, CP, 8:10). Así como la detección de errores, "porque sirven al alumno para detectar los errores cometidos en su interpretación" (D32, $\mathrm{CM}, 25: 8$ ). "Como dije, con estas grabaciones los alumnos se dan cuenta de los fallos cometidos en la obra interpretada por lo que les resultan muy útiles para mejorar con mayor rapidez" (D32, CM, 25:9).

También es un importante soporte que permite hacer cambios después de la escucha. El proceso de realizar una grabación favorece la formación del discente al ser un medio de perfeccionamiento de la obra interpretada, "obliga a un estudio más consciente y cuidado" (D11, CP, 3:12) y "les obliga a realizar un trabajo extra de estudio para perfeccionar la obra" (D35, EM, 28:9). "Si la grabación es externa, proporciona otra visión musical de lo que se está trabajando" (D40, EM, 34:10).

Otro de los beneficios es que ayuda en la mejora del rendimiento al alumnado en la interpretación musical porque "cuando un músico participa en una grabación, tanto alumnos como profesionales, supone un reto, por lo cual va a intentar dar lo mejor de sí mismo, por lo que se esforzará para conseguir los mejores resultados posibles" (D21, CM, 13:13). Así mismo "las grabaciones suponen una motivación por parte del mismo, con lo cual mejora su rendimiento" (D25, CM, 17:8).

Para concluir, la grabación se convierte en un soporte que ayuda a alcanzar la calidad de la interpretación, "siempre que se cumplan los criterios de calidad, en cuanto al proyecto se refiere, como evaluaciones tanto 
internas como externas" (D33, CM, 26:8), "dado que tenemos que generar en el alumno la importancia de realizar un trabajo de calidad, para el cual es indispensable una evaluación democrática" (D33, CM, 26:9). Resultando imprescindible su uso "tanto en la práctica instrumental como en otras materias como puede ser composición, donde los alumnos podrían disponer de grabaciones de calidad de sus trabajos" (D15, CP, 7.11).

Cabe señalar que a pesar de la total libertad con que los entrevistados han expresado sus opiniones sobre las grabaciones, y que no han tenido contacto entre ellos, ninguno de los 41 ha destacado ningún aspecto negativo de las mismas, refiriendo sólo sus aspectos positivos.

\section{Discusión}

A lo largo de este trabajo se ha evidenciado que la sociedad actual requiere un uso generalizado de las TIC en todos los ámbitos, lo cual no se puede obviar en el educativo, donde se convierten en un importante soporte para el docente. En una realidad educativa en la que el discente ya no es un mero receptor de información, sino el protagonista de su proceso de aprendizaje, se hace necesario manejar recursos que favorezcan sus habilidades. Y las TIC constituyen una poderosa estrategia didáctica, poco utilizada, sin embargo, en el aprendizaje musical.

Atendiendo a los resultados se puede concluir que una de las mayores aportaciones de las TIC a la formación musical son las grabaciones digitales, que resultan un excelente recurso, no sólo como modelo a seguir de interpretaciones de grandes virtuosos, sino como herramienta de autocorrección cuando es el mismo estudiante quien realiza la grabación de su interpretación. Además, ésta se convierte en un instrumento de apoyo del estudio técnico, sonoro e interpretativo, y posibilita mejorar la autoconsciencia, la autocrítica y autoevaluación de la propia exposición auditiva. Es obvio que en la formación musical es necesario promover prácticas que fomenten la autoescucha, el auto examinarse, la autocorrección (donde el alumnado hace un análisis del logro de sus objetivos y competencias), y en este sentido la grabación le permite hacer una valoración personal sobre si el proceso de aprendizaje está o no teniendo éxito y con ello generar un aprendizaje autónomo, lo cual supone un excelente recurso motivacional. El vivenciar de primera mano cómo se percibe su interpretación resulta un acicate motivador de la práctica. Un aprendizaje que no debe limitarse al aula, sino que impregne al alumnado con destrezas que pueda beneficiarlo en sus futuras actividades profesionales e interpersonales.

Por último, se puede concluir que la exigencia y perfeccionamiento que supone trabajar una obra implica para el discente un importante nivel de esfuerzo en la interpretación musical, teniendo como meta obtener la máxima calidad de la obra. Las grabaciones suponen, por tanto, un valioso recurso para alcanzar este objetivo y obtener un mayor rendimiento y una mejora de la práctica musical. No obstante, es necesario señalar que las grabaciones podrían suponer una causa de desigualdad en aquellos estudiantes que no cuenten con recursos para poder realizarlas. Asimismo, entrañan una dificultad añadida, al requerir un manejo mínimo de medios informáticos, por lo que tanto algún docente como discente podría verlo como un obstáculo. Además, podrían constituir un recurso contraproducente si la persona que las emplea posteriormente no tiene el feedback de alguien que le redirija, matice o corrija sus fallos.

Todo lo expuesto avala la importancia de los hallazgos aquí presentados, máxime en un ámbito en los que hay una evidente ausencia de investigaciones que visibilicen la importancia y necesidad de su empleo en el aula.

Finalmente, como líneas de futuro, este estudio se complementará con la visión de los estudiantes, y se inquirirá de nuevo a los docentes para conocer su opinión sobre la actual situación educativa derivada del coronavirus, que ha planteado nuevas posibilidades en la educación musical, así como sobre las problemáticas que pudiesen derivarse de las grabaciones.

\section{Referencias}

Adell, J. y Castañeda L. (2012). Tecnologías emergentes, ¿pedagogías emergentes? En J. Hernández, M., Pennesi, D. Sobrino y A. Vázquez (Coord.), Tendencias emergentes en educación con TIC (pp. 13-32). Barcelona: Asociación Esperial, Educación y Tecnología. 
Area, M. y Ribeiro, M.T. (2012). De lo sólido a lo líquido: Las nuevas alfabetizaciones ante los cambios culturales de la Web 2.0 [From Solid to Liquid: New Literacies to the Cultural Changes of Web 2.0]. Comunicar, 38, 13-20. https://doi.org/10.3916/C38-2012-02-01

Arques, R. y Navas, L. (2012). Las actitudes de los docentes de música ante las TIC. International Journal of Developmental and Educational Psychology, 1(4), 403-410. Disponible en: https://www.redalyc.org/ articulo.oa? id=349832337043

Belloch, C. (2012). Las tecnologías de la Información y Comunicación en el aprendizaje. Material docente [on-line]. Departamento de Métodos de Investigación y Diagnóstico en Educación. Universidad de Valencia. Disponible en: https://www.uv.es/bellochc/pedagogia/EVA1.pdf

Brendel, A. (2013). De la A a la Z de un pianista. Un libro para amantes del piano. Barcelona: Acantilado.

Cabero, J. y Barroso, J. (2013). La utilización del juicio de experto para la evaluación de TIC: el coeficiente de competencia experta, Bordón, 65(2), 25-38. https://recyt.fecyt.es/index.php/BORDON/article/view/ brp.2013.65202

Carrascosa, R. y Ruiz-Silva, J. C. (1977). Pro y contra: artistas a favor del disco, artistas en contra del disco. Ritmo, 48(477), 119-124.

Carrera, E., Román, S. y Chao-Fernández, R. (2017). Free software in music education: an interdisciplinary practical approach in Primary School. En J. M. Dodero, M. Ibarra e I. Ruiz (Coords.). Fifth International Conference on Technological Ecosystems for Enhancing Multiculturality (pp.1-7). New York: ACM Digital Library. https://doi.org/10.1145/3144826.3145428

Chao-Fernández, A., Pérez Crego, M. C. y García Armas, N. J. (2019). Percepción del profesorado de los conservatorios de Galicia sobre las grabaciones de la compañía de la Radio Televisión de Galicia (CRTVG), en T. Sola et al (eds.), Innovación educativa en la sociedad actual (pp. 2003-2014). Madrid: Dykinson.

Clarke, E. (2006). Escuchar la interpretación, en Rink, J (ed.). La interpretación musical. Madrid: Alianza Editorial.

De Castro, C. (2015). Recursos educativos TIC en la enseñanza musical pianística. Revista Electrónica Complutense de Investigación en Educación Musical, 12, 37-52. https://doi.org/10.5209/rev_RECIEM. 2015.v12.49004

Denzin, N. K. (1989). The Research Act. Englewood Cliffs, NJ: Prentice Hall.

Díez, N. y Carrera, X. (2018). Integración de las TIC en los procesos de enseñanza-aprendizaje de la especialidad de Pedagogía en los conservatorios superiores de música. Revista interuniversitaria de Investigación en Tecnología Educativa, 5, 40-55. https://doi.org/10.6018/riite/2018/342681

Ferro, C., Martínez, A. y Otero, M. (2009). Ventajas del uso de las TICs en el proceso de enseñanza-aprendizaje desde la óptica de los docentes universitarios españoles. EDUTEC. https://doi.org/10.21556/edutec. 2009.29.451

Flick, U. (2004). Introducción a la investigación cualitativa. Madrid: Ediciones Morata.

Gértrudix Barrio, M. (2003). Música, narración y medios audiovisuales. Madrid: Ediciones del Laberinto.

Gisbert Caudeli, V. (2018). La creatividad musical como herramienta educativa para el cambio social. Creatividad y Sociedad, 27, 26-46. http://creatividadysociedad.com/wp-admin/Art\%C3\%ADculos/27/2. La\%20creatividad\%20musical\%20como\%20herramienta\%20educativa\%20para\%20el\%20cambio\%20soc ial.pdf?_t=1576012033

Goetz, J. y LeCompte, M. (1998). Etnografía y diseño cualitativo en investigación educativa. Madrid: Ediciones Morata.

Marqués, P. (2012). Impacto de las TIC en la educación: funciones y limitaciones. 3 c TIC: cuadernos de desarrollo aplicados a las TIC. 2(1), 1-15. https://doi.org/10.17993/3ctic.2013.21.\%20

Miles, Huberman, M. y Saldaña, J. (2014). Qualitative Data Analysis. A Methods Sourcebook. London: Sage. Miles, M. \& Huberman, M. (1994). Qualitative Data Analysis. An expanded sourcebook. California: Sage.

Ministerio de Educación, Cultura y Deporte (2001). Los desafíos de las Tecnologías de la Información y las Comunicaciones en la Educación. España: OCDE. 
Miranda Regojo, F. (1990). La fonoteca. Madrid, Pirámide.

Pérez Sánchez, A. (2012). El legado sonoro de Iberia de Isaac Albéniz. La grabación integral: un estudio de caso. Tesis doctoral, Facultad de Geografía e Historia, Departamento de Musicología, leída el 29-06-2012. Universidad Complutense de Madrid. https://eprints.ucm.es/21838/

Pujadas, J. (2000). El método biográfico y los géneros de la memoria. Revista de antropología social, 9,127158. https://revistas.ucm.es/index.php/RASO/article/view/RASO0000110127A

Román-García, S., Chao-Fernández, R. y Mato-Vázquez, D. (2018). La PDI como facilitadora del aprendizaje de música y matemáticas en Educación Infantil. En E. Martínez Rodríguez (Coord.), Aportaciones de vanguardia en la investigación actual (pp. 363-376). Madrid: TECNOS.

Stake, R. (1998). Investigación con estudio de casos. Madrid. Morata.

Valcárcel, A. y González, L. (2013). Uso pedagógico de materiales y recursos educativos de las TIC: sus ventajas en el aula. Departamento de Didáctica, Organización y Métodos de Investigación. Universidad de Salamanca. Disponible en: http://postitulo.secundaria.infd.edu.ar/archivos/repositorio/1000/1111/USala manca_Recursos_TIC.pdf

Fecha de recepción: 6 de mayo de 2020.

Fecha de revisión: 8 de junio de 2020 . Fecha de aceptación: 15 de junio de 2020. Fecha de publicación: 1 de julio de 2020. 